\title{
Faire discipline : la géographie à la Société de Géographie de Paris entre 1800 et 1850
}

\section{Laura Péaud}

\section{(2) OpenEdition}

\section{Journals}

Édition électronique

URL : https://journals.openedition.org/cdg/1507

DOI : $10.4000 /$ cdg. 1507

ISSN : 2107-7266

Éditeur

UMR 245 - CESSMA

\section{Référence électronique}

Laura Péaud, «Faire discipline : la géographie à la Société de Géographie de Paris entre 1800 et 1850 », Carnets de géographes [En ligne], 11 | 2018, mis en ligne le 15 septembre 2018, consulté le 20 mai 2021. URL : http://journals.openedition.org/cdg/1507 ; DOI : https://doi.org/10.4000/cdg.1507

Ce document a été généré automatiquement le 20 mai 2021.

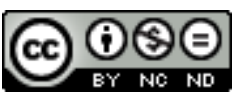

La revue Carnets de géographes est mise à disposition selon les termes de la Licence Creative Commons Attribution - Pas d'Utilisation Commerciale - Pas de Modification 4.0 International. 


\title{
Faire discipline : la géographie à la Société de Géographie de Paris entre 1800 et 1850
}

\author{
Laura Péaud
}

1 À partir du début du XIXe siècle, plusieurs figures scientifiques promeuvent la géographie et font l'article pour qu'elle soit reconnue comme une discipline à part entière. En France, c'est en particulier le cas de Conrad-Malte-Brun, géographe danois exilé à Paris (Godlewska, 1991), qui en 1807 déclare :

2 «Enfin, il est temps qu'à l'exemple de l'Histoire naturelle, de la Chimie et de la Médecine, les Sciences Géographiques possèdent un dépôt où les hommes voués à ce genre d'études, puissent consigner en commun des travaux qui tendent au même but, discuter les difficultés qui les arrêtent, faire un échange continuel de lumières et de découvertes, et surtout répandre de plus en plus le goût de ces connaissances [...].» (Malte-Brun, $1807: 7$ ).

3 L'enthousiasme de Malte-Brun correspond à un moment de premières "naissances " européennes de la géographie, qui se traduit par une pluralité de projets pour une discipline géographique(Besse, Blais et Surun, 2010; Blais et Laboulais, 2006). De semblables appels résonnent en Prusse, sous l'impulsion de Carl Ritter notamment, nommé à partir de 1820 à la chaire de géographie de l'Université de Berlin, qui contribue à la création de la société de géographie de Berlin en 1828. Même si, en France, ce premier élan disciplinaire peut paraître incertain, hésitant et, par certains aspects, bancal, au regard de ce que sera par la suite l'école française de géographie de la fin du siècle, un certain nombre de points d'ancrage dessinent une envie disciplinaire. Trois ressorts fondamentaux semblent soutenir l'existence disciplinaire de la géographie : la création de nouveaux lieux de savoir devant assurer une visibilité publique et une place dans le paysage scientifique français et parisien, le renforcement d'une cohésion disciplinaire par l'identification de figures géographiques, ainsi que la normalisation progressive des gestes et des cadres de l'écriture géographique. Ces deux dernières préoccupations font l'objet de discussions de plus en plus récurrentes et 
importantes, mais ne donnent cependant pas lieu à des réponses fermes et stabilisées au milieu du XIXe siècle, qui permettraient d'identifier un véritable "programme scientifique » (Lakatos, 1994).

4 Cet article vise donc à mettre en avant les éléments saillants du projet disciplinaire qui se dessine en France pour la géographie au début du XIXe siècle, c'est-à-dire les points d'accord à partir desquels les nouveaux «géographes » bâtissent un projet disciplinaire. L'argumentaire se fonde sur des sources textuelles variées (correspondances, bulletins, ouvrages et revues) issues de l'activité de la Société de Géographie de Paris, à l'initiativede ce "faire discipline». Le propos s'appuie sur le parcours d'une figure emblématique de ce moment, celle d'Edme-François Jomard. Il s'agit ici de mettre en évidence les jalons de cette première tentative disciplinaire pour la géographie en France ${ }^{1}$, portée essentiellement par la Société de Géographie de Paris, sans éluder pour autant les difficultés voire les contradictions que ce projet porte en lui. Car si les géographes manifestent bien une envie disciplinaire, leur projet se résume essentiellement à assurer une visibilité et une reconnaissance publique à la géographie plus qu'à fonder réellement un solide programme scientifique soutenant la discipline.

Nous verrons tout d'abord que la volonté de "faire discipline " s'incarne spatialement, par la création de lieux pour les géographes. Ceux-ci (se) cherchent ensuite des figures de référence de la discipline, dans le passé et le présent, pour mieux asseoir leur projet. Enfin, malgré ces premiers jalons d'institutionnalisation, la création d'une discipline géographique achoppe sur une théorisation scientifique encore peu développée.

\section{Créer de nouveaux lieux pour les géographes et le savoir géographique}

6 La première étape du processus de création disciplinaire consiste en une incarnation de cette volonté dans des lieux, c'est-à-dire une mise en visibilité de la géographie.

\section{La fondation des lieux d'un collectif disciplinaire en devenir}

7 Pour répondre à cette ambition de faire dela géographie une discipline reconnue, plusieurs sociétés de géographie sont presque simultanément créées en France, Prusse et Grande-Bretagne : en 1821 à Paris, 1828 à Berlin et 1830 à Londres. Baliser et étendre la géographie des savoirs géographiques, assurer et pérenniser la matérialité des savoirs géographiques, tels pourraient être, en résumé, les objectifs communs des trois premières sociétés. Le statut de la Société de Géographie de Paris l'indique très clairement :

«Article 1: La Société est instituée pour concourir aux progrès de la Géographie; elle fait entreprendre des Voyages dans les contrées inconnues; elle propose et décerne des prix; établit une correspondance avec les Sociétés savantes, les Voyageurs et les Géographes; publie des relations inédites ainsi que des ouvrages, et fait graver des cartes. » (Bulletin de la Société de Géographie de Paris², 1822, S1, T1 : 3)

Cette fondation n'a pas qu'un pur objectif scientifique, elle possède un caractère performatif, puisqu'elle a la charge de rendre visible un nouveau champ disciplinaire. Sa situation spatiale et symbolique importe donc autant que l'objectif qu'il lui est assigné. La société parisienne s'installe ainsi dès l'origine au centre de Paris, dans le 
Quartier latin ou dans son immédiate proximité. Elle occupe successivement des locaux au 12 rue Taranne (1821), au 36 passage Dauphine (1827), au 23 rue de l'Université (1833) puis au 3 rue Christine (1853). Encore aujourd'hui, la société se situe dans une position centrale par rapport aux lieux du savoir, puisqu'elle occupe au 184 boulevard Saint-Germain un bâtiment dédié depuis 1878. Avant cette date, puis à partir du moment où la charte de la Société de Paris est approuvée par le roi en 1827, elle dispose de quelques facilitations matérielles. La société est en effet déclarée d'utilité publique et voit converger les bonnes volontés vers elle, en particulier celles des ministres de Louis XVIII qui font partie de ses membres(Lejeune, 1993). Cette reconnaissance politique accentue bien sûr sa reconnaissance publique et celle des ambitions portées par ses membres.

10 Même si la Société de Géographie de Paris affiche dès sa fondation des ambitions scientifiques très claires, il ne faudrait pas oublier qu'elle fonctionne aussi, et peut-être avant tout, comme un lieu de sociabilité. L'envie de créer un lieu dédié à la discipline géographique répond aussi à un désir d'entre-soi. Comme Malte-Brun s'en fait l'écho dès 1807 , faire discipline consiste à rassembler des individus qui partagent cette volonté et se reconnaissent comme la partageant :

11 "Nous avons l'espérance certaine que les Arrowsmith, les Barbié du Bocage, les Bougainville, les Bourgoing, les Brédow, les Brunss, les Buache, les Choiseul, les Corréa, les Darlymple, les Deguigne, les Dusheil, les Ebert, les Fabri, les Fleurieu, les Forestier, les Gaspari, les Gosselin, les Heeren, les Humboldt, les Jacotin, les Langlès, les Lapie, les Larcher, les Lévêque, les Lichstenstern, les Lowenorn, les Mannert, les Mentelle, les Meusel, les Millin, les Münster, les Olivier, les Péron, les Rosily, les Sainte-Croix, les Sacy, les Sanson, les Sonnini, les Volney, les Walckenaer, les Weyland, les Zach, les Zimmermann, et en un mot, tous ceux qui cultivent l'histoire et la géographie, contribueront, les uns par leurs conseils et leurs suffrages, les autres par leurs talens [sic] et leurs travaux [...]. » (Malte-Brun, $1807:$ : 8-9).

12 Faire discipline signifie ici clairement faire corps (Godlewska, 1999), c'est-à-dire se reconnaître soi-même tout en assurant une visibilité vers l'extérieur. Ces figures sont par ailleurs liées par une habitude de sociabilité (banquets, discussions informelles, autres activités sociales) autant que lepartage d'ambitions scientifiques communes (Laboulais-Lesage, 2005).

\section{Quels savoirs géographiques hors de la Société de Géographie?}

13 À côté de la société de géographie, pensée comme le lieu de convergence structurant le collectif géographique, d'autres lieux participent à l'entreprise disciplinaire. Il s'agit notamment des bibliothèques et cartothèques, pensées comme les dépôts des sociétés de géographie, ainsi que des lieux de vulgarisation du savoir, tels que les géoramas ${ }^{3}$ qui remportent d'importants succès en suscitant l'enthousiasme du public (Besse, 2003). Si les partisans d'une géographie institutionnalisée s'investissent ponctuellement dans des projets destinés au grand public, leurs efforts se concentrent cependantsur des entreprises d'institutionnalisation du savoir géographique.

Parmi ces lieux, le projet de bibliothèque géographique soutenu ardemment par EdmeFrançois Jomard (1777-1862) dès les années 1820 est emblématique en même temps qu'il reflète le caractère incertain de la reconnaissance disciplinaire de la géographie. En 1828, Jomard obtient du ministre Martignac d'ériger le Dépôt de géographie de la 
Bibliothèque Royale en cinquième département, dit « des cartes géographiques ». Cela lui confère évidemment plus d'indépendance et la possibilité de travailler en collaboration avec la Société de Géographie. Mais certains commissaires qui enquêtent sur la Bibliothèque, au sein d'une commission fondée à cet effet, sont assez critiques quant à ses actions. Jomard essaie de prévenir les attaques des autres directeurs de département sur la légitimité et l'indépendance du sien (Jomard, 1831), mais le ministère propose de replacer les cartes dans le département des livres et imprimés. En 1832, la bibliothèque passant du ministère de l'Intérieur à celui de l'Instruction publique, le ministre Guizot supprime le dépôt des cartes et le rattache à celui des estampes. À partir de ce moment, Jomard mène une lutte incessante pour reconquérir l'indépendance perdue du dépôt. Il présenteune nouvelle brochureen 1848, alors qu'il pressent de nouveaux bouleversements politiques, sous le titre De la collection créée à la Bibliothèque royale. Examen de ce qu'on a fait et de ce qui reste à faire pour compléter cette création et la rendre digne de la France. Il y défend, comme il le fait depuis plus de vingt ans, l'utilité et la nécessité d'un fonds géographique indépendant (Jomard, 1848) et inscrit cet objectif dans le processus général de structuration de la discipline géographique. Ses vœux semblent enfin entendus, puisqu'en 1854, le ministre Fortoul ressuscite le département, en soulignant qu'il veut en faire la base d'un musée d'ethnographie et des voyages. Mais de nouveau, le département est rattaché aux Estampes en 1858, à la grande fureur de Jomard, à qui l'on répond qu'on ne sépare pas la géographie de l'histoire. Il faudra attendre 1942, malgré tous les efforts déployés par Cortambert son successeur, pour voir le département autonome. L'obstination de Jomard symbolise ici la volonté d'une autonomisation assurée de la géographie, qui mérite d'exister en elle-même, alors que les obstacles opposés à un dépôt indépendant rappellent la situation de dépendance dans laquelle se situe la géographie, notamment vis-à-vis de l'histoire.

En dehors de ces quelques lieux emblématiques, au statut néanmoins incertain, l'incarnation institutionnelle des savoirs géographiques demeure restreinte, à l'exception du Dépôt de la Guerre, lieu clé en matière de production de savoirs géographiques militaires (Bret, 1991). En revanche, du côté de l'enseignement de la géographie, les lieux se réduisent considérablement. A la suite de la fermeture de l'éphémère École Normale Supérieure, où Edme Mentelle et Jean-Philippe Buache de la Neuville ont dispensé des cours, la géographie disparaît du système d'enseignement (Nordman, 1994). La discipline n'est enseignée que ponctuellement, se confondant le plus souvent avec la cartographie et l'ingénierie militaire (Bret, 1991), jusqu'à la fin du XIXesiècle avant que les réformes de Levasseur n'en fassent un enseignement obligatoire au primaire (Lefort, 1990) et que les premières chaires universitaires apparaissent ${ }^{4}$. Cette absence de lieux d'enseignement limite considérablement les efforts contenus dans cette première institutionnalisation. En comparaison, la situation prussienne est plus favorable à l'institutionnalisation précoce de la géographie : Carl Ritter occupe en effet une chaire à la fois à l'Académie militaire et à l'Université de Berlin dès 1820 , ce qui contribue à une visibilité plus grande de la discipline (Lüdecke, 2002). De même, la régionalisation s'opère de manière tardive en France, puisqu'il faut attendre les années 1870 pour que les premières sociétés de géographie apparaissent hors de Paris (Lejeune, 1993). 


\section{Identifier les figures géographiques de référence}

Incarner la géographie dans des lieux ne suffit pas à assurer son existence et sa légitimité disciplinaire. Il faut également que lui soient associées des figures, dans le présent et le passé, permettant d'assurer la mémoire et la validité de l'édifice disciplinaire.

\section{Vers plus d'exclusivité de la pratique géographique}

L'édification de la société de géographie de Paris marque le besoin de consacrer non seulement un lieu et du temps aux savoirs géographiques, mais aussi des individus, qui reconnaissent l'opportunité de développer cette branche. Tous les membres ${ }^{5}$ ne se dévouent pas entièrement à cette entreprise, loin s'en faut, puisqu'une grande partie revendique encore un esprit encyclopédiste, amateur et passionné par le progrès des sciences en général, à l'instar d'Alexander von Humboldt par exemple; mais la construction de lieux dédiés à ce champ répond aussi à une tendance à l'exclusivité de la pratique géographique et permet de la soutenir.

18 Edme-François Jomard représente un bon exemple de cette tendance. Faisant partie de la première génération de savants formée sous les auspices révolutionnaires (Laissus, 2004) et dans la tradition d'une géographie militaire (Bret, 1991), il participe à ce mouvement de spécialisation disciplinaire. Jomard suit des cours du Nouveau Muséum d'Histoire Naturelle que la Convention a substitué en 1793 au vieux Jardin du Roi. En 1794, il entre ensuite à l'École des Ponts et Chaussées de Paris ; déçu, Jomard demande ensuite à rejoindre la nouvelle École centrale des travaux publics, la future École polytechnique créée en 1794 à l'instigation de Monge et Lamblardie. Cinq catégories d'ingénieurs y sont formées : ingénieurs militaires, ingénieurs des ponts et chaussées, ingénieurs géographes, ingénieurs des mines et ingénieurs constructeurs de vaisseaux. Jomard choisit la troisième catégorie, alors même que cette voie n'est en rien solide, comme l'explique Yves Laissus: «Au moment, en effet, où il décide d'embrasser la carrière d'ingénieur géographe, le statut de cette profession paraît mal assuré, disputée qu'elle est entre civils et militaires. » (Ibid. : 33). Ce choix initial structure ensuite toute sa carrière, car Jomard ne se départit jamais de son intérêt pour la géographie. Il y entre par la voie professionnalisante et la dimension militaire, alors classique, en faisant ses armes d'ingénieur géographe sur le terrain égyptien (1798-1802) en compagnie de l'escadron de savants escortant Bonaparte, puis passe progressivement à l'animation de la discipline naissante (Bret, 2003). Le tournant s'opère véritablement alors que la Description d'Égypte touche à sa fin. À partir des années 1820, Jomard se lance alors à corps perdu dans la défense des intérêts géographiques (Laissus, 2004). Il fait partie des membres fondateurs de la Société de Géographie de Paris, entretient des réseaux de correspondance denses pour alimenter les séances et les bulletins et s'institue spécialiste ès Afrique. L'Égypte devient son terrain de prédilection et il joue un rôle plus qu'actif dans l'affaire René Caillé en $1828^{6}$. Il se distingue par son rôle d'animateur hors pair des débats géographiques, et par sa défense inlassable des intérêts de la toute récente discipline, notamment à travers ses actions répétées en faveur d'une bibliothèque et d'une cartothèque dédiées aux savoirs géographiques, et plus généralement par son enthousiasme pour tout projet permettant une plus grande 
visibilité de ces savoirs. Jomard s'engage avec un engouement réel et son parcours constitue, de fait, l'une des premières carrières entièrement consacrées à ce champ.

$\mathrm{Au}$ milieu du XIXe siècle, bien peu ont alors opéré un tel tournant en faveur d'une discipline et en particulier de la géographie. Jomard fait en la matière figure d'exception et ne trouve son pendant qu'en Prusse, en la personne de Carl Ritter (Beck, 1979). En France, il faut attendre les années 1860 avant que ne s'affirment et s'affichent des carrières de géographes; avec par exemple Reclus ou Vivien de Saint-Martin pour les plus célèbres d'entre eux.

\section{L'invention d'une histoire de la géographie}

Plus efficacement que l'exclusivité de la pratique géographique, encore marginale, la cohésion disciplinaire passe par la (re)découverte et l'écriture de l'histoire de la géographie, via l'identification des héros d'un passé disciplinaire répondant aux besoins des géographes du XIXesiècle. Alors que les États européens se cherchent des génies nationaux (Thiesse, 1999), les géographes entreprennent eux aussi de célébrer leurs « monuments » et héros.

Cette tendance se traduit par quelques entreprises emblématiques, comme celle de Jomard, toujours lui, qui se propose de compiler une mémoire cartographique de la discipline, comme pour prouver de son antériorité et donc de sa légitimité historique à exister au début du XIXe siècle. À partir de 1828, il débute la réalisation d'un ouvrage d'envergure, les Monuments de la géographie, qui doit compiler les cartes les plus importantes dans l'histoire de la géographie. Ici, géographie et cartographie sont nettement confondues. Il met plusieurs années à les publier : quelques planches sont gravées en 1842, mais il faut attendre 1862 pour que la vingtaine de planches initialement prévues paraissent, et 1879 pour qu'Eugène Cortambert écrive une introduction à cet ouvrage qui parait sans texte à l'origine (Laissus, 2004). Dans l'édition de 1862, Jomard insère cependant une petite note préliminaire, dans laquelle il revient sur l'esprit de cette entreprise :

«[...] persuadé de l'utilité qu'il pouvait y avoir, pour les savants, pour les historiens, pour les amis des études géographiques, d'avoir un recueil de cartes fac-simile, où les découvertes géographiques seraient consignées de la manière la plus authentique, et, par-là, les titres des peuples navigateurs mis, pour ainsi dire, au-dessus de toute discussion; convaincu de l'avantage qu'il y aurait pour les hommes de cabinet de pouvoir consulter, sans déplacement, des monuments totalement ignorés, ou très-peu connus, dispersés dans l'Europe entière en trente endroits différents; pensant, enfin, qu'une telle collection serait, en quelque sorte, une histoire de la Géographie écrite par elle-même, nous ne ménagions ni soins, ni correspondances, ni voyages dispendieux, afin de nous mettre en état de remplir cette tâche d'une manière satisfaisante pour le public [...]. » (Jomard, 1862 : NP)

L'objectif de Jomard correspond donc à la mise à disposition pour le collectif géographique d'une compilation cartographique des plus grands travaux de la discipline. La liste des 21 planches publiées montre que Jomard a arpenté tous les terrains européens, ainsi que le bassin méditerranéen, mais en se concentrant sur la période du haut Moyen Âge et de la Renaissance (du XIeau XVIe siècle).

Outre cette entreprise particulière, les bulletins de la société parisienne renferment également nombre d'articles ou de notices portant sur les grands classiques de la 
discipline. Ce sont généralement toujours les mêmes références qui reviennent, d'une manière très consensuelle. Ainsi, Hérodote, Strabon ou encore Ptolémée sont-ils régulièrement mobilisés pour l'Antiquité. Du côté du MoyenÂge, ce sont les auteurs arabes que l'on redécouvre: en 1839, la société parisienne annonce par exemple la réédition des travaux d'El-Idrisi (BSGP, 1839, S2, T11) pour lesquels elle se passionne ${ }^{7}$. Mais ce sont surtout les héros de la Renaissance que l'on célèbre, les navigateurs ayant ouvert la porte du nouveau monde et permis le renouveau de l'exploration. Une coïncidence se manifeste ici clairement entre géographie et exploration, Christophe Colomb apparaissant en particulier comme la figure mythique de la géographie. Il trouve un fervent partisan en la personne d'Alexander von Humboldt, qui lui consacre une Histoire de la géographie du nouveau continent (1836-1837). En dépit du titre généraliste, c'est bien la figure de Colomb qui est présentée majoritairement. À travers ce livre, Humboldt vise deux objectifs. D'abord, il cherche à retracer le fil des recherches géographiques, à tracer une continuité historique de la discipline, pour lui en assurer des bases aussi anciennes que solides. L'idée de continuité rejoint la volonté de faire discipline, en prouvant l'existence d'une épaisseur temporelle conséquente qui viendrait soutenir la volonté de faire discipline. On retrouve chez Humboldt le socle de la Renaissance tant vanté par Jomard dans ses Monuments de la géographie et qui semble constituer l'âge d'or auquel la géographie du XIXe siècle se réfère. Le quinzième siècle en particulier fascine, c'est-à-dire le temps des grandes découvertes et de la dilatation géographique du monde (Grataloup, 2007). Peut-être les géographes du XIXe siècle y trouvent-ils un certain parallélisme avec le double mouvement de conquête coloniale et de révolution des transports alors à l'œuvre, qui participe aussi à l'ouverture de l'horizon européen et au changement de grandeur de la terre (Besse, 2003) ?Ces entreprises servent, dans un effet miroir et réflexif, à éclairer le moment moderne de la géographie d'exploration pour mieux légitimer le temps d'institutionnalisation de la discipline au début du XIXe siècle ${ }^{8}$.

Cette entreprise de l'histoire de la géographie met très largement de côté la géographie militaire, dont les apports sont pourtant considérables à la discipline historiquement mais également pour la période considérée, notamment au sein du Dépôt de la Guerre. De la même manière, aucune critique ne vient vraiment émailler le portrait des héros mentionnés : aucun des explorateurs mythiques ne voit en effet son image écornée. La réflexivité des géographes du XIXe siècle se trouve donc encadrée par le désir de reconnaissance disciplinaire.

Il est également intéressant de noter que ces entreprises d'écriture de l'histoire d'une discipline naissante donnent lieu à des tensions entre les acteurs de la géographie, liées à des enjeux de primauté ou suprématie nationale. Les Monuments de Jomard rencontre par exemple la concurrence du Vicomte de Santarem, qui dès 1842 fait paraître à Paris ses Recherches sur la priorité de la découverte des pays situés sur la côte Occidentale d'Afrique, au-delà du cap Bojador, et sur les progrès de la science géographique, après les navigations des portugais au XVe siècle, accompagnées d'un atlas composé de mappemondes, et de cartes pour le plupart inédites, dressées depuis le XIe siècle jusqu'au XVIIe siècle (1842), perçue comme une vraie menace, d'autant plus qu'il se positionne sur le même créneau temporel.

27 Lors de la séance du 4 mars 1842, Jomard s'offre un droit de réponse à la Société de géographie de Paris, dans laquelle il affirme sa primauté intellectuelle quant à ce projet : 
«Personne, dit M. Santarem, n'avait conçu cette idée ; ainsi l'auteur de cette singulière assertion oublie que la pensée de publier les anciennes cartes est émanée au sein de la Société de Géographie, quinze ans au moins auparavant; il perd de vue sa propre déclaration, qui date de plus de cinq ans. En effet, quand il communiqua, en 1842, les premières feuilles gravées de ses cartes du moyen âge, sans dire un seul mot de ceux qui avaient travaillé avant lui sur ce sujet, une vive réclamation fut faite immédiatement, et alors il déclara que loin de contester à $\mathrm{M}$. Jomard la priorité de ses projets de publication il mentionnait lui-même, dans le volume destiné à accompagner son atlas, les travaux de son collègue (BSGP, 1842, S2, T22 : 4-5)

L'affaire pourrait être interprétée comme une simple anecdote, mais elle reflète les tensions à l'œuvre dans l'écriture de l'histoire de la discipline naissante, profondément inscrite dans les jeux de concurrence que se livrent les nations européennes. Dans ses ouvrages, Santarem vante ainsi la primauté de la nation portugaise en termes de découvertes et se fait ainsi le champion de son pays. Écrire l'histoire de la géographie ne se réduit donc pas à prendre connaissance d'un passé commun, mais permet d'inscrire dans le marbre les suprématiesnationales si prégnantes dans le milieu du XIXe siècle. C'est bien d'ailleurs le propre de toute historiographie, que d'aller au-delà d'une simple convocation du passé : celui-ci n'est jamais mobilisé par hasard, et la confrontation des entreprises de Jomard et de Santarem montre bien le caractère nécessairement partial de ce genre d'entreprises. Dans ce cas, l'écriture de l'histoire de la géographie balbutiante visa à la fois à assurer les bases de la discipline, mais des bases qui tendent à puiser leurs racines dans des récits nationaux.

\section{Écrire géographiquement le monde?}

Même si l'on ne peut clairement identifier les modalités d'une écriture géographique, car les géographes sont davantage occupés à assurer une visibilité à la discipline qu'à fonder un vrai programme scientifique (Lakatos, 1994), une réflexion en germe porte néanmoins sur le régime de scientificité que la géographie doit adopter. Un des principaux aspects de cette discussion porte sur la fixation de la pratique géographique, en particulier les gestes, et sur les conditions d'une écriture scientifique de la géographie, celle-ci devant se faire administration de la preuve.

\section{«Scientificiser » les gestes et l'écriture géographique}

31 Parvenir à une scientificité accrue des savoirs géographiques et à leur inscription dans le champ des sciences expérimentales, donc irréfutables, constitue l'objectif de ces réflexions balbutiantes. Un premier effort doit porter sur la professionnalisation des gestes géographiques, à travers à la fois l'instrumentation généralisée de la pratique et son encadrement. Cette dimension instrumentale de la science n'est pas nouvelle (Bourguet, Licoppe, Sibum, 2002), mais s'amplifie alors que les géographes veulent assurer des bases scientifiques à leur discipline. Cela se traduit d'une part par une attention de tous les instants aux outils utilisés. Les bulletins des sociétés regorgent d'articles indiquant les nouvelles observations réalisées ici ou là et se font ainsi l'écho de cette préoccupation générale. Dès le premier bulletin de la société parisienne, $\mathrm{M}$. Cailliaud présente par exemple un article sur les progrès faits grâce à l'utilisation de l'aiguille aimantée (BSGP, 1822, S1, T1) ; la revue consacre dans ses premiers numéros 
une section spéciale dédiée aux instruments et aux procédés, qui recense les améliorations apportées aux instruments. En 1825, il est par exemple question du perfectionnement de la boussole en lui appliquant une plaque de cuivre, réalisé par $\mathrm{M}$. Arago, et de ses conséquences sur le travail géographique :

«Elle hâte et précise le travail dans les opérations de terre; elle donne en mer le moyen de diminuer les oscillations toujours reproduites par les mouvements du vaisseau; et comme le savant inventeur est parvenu même à calculer l'action du cuivre sur l'aiguille, de manière à reconnaître une déviation de moins de quelques secondes, on pourra désormais se garantir des erreurs que l'on commettait nécessairement lorsque, pour évaluer l'intensité magnétique de la terre, on avait recours à des boussoles dans lesquelles l'aiguille était entourée d'un cercle de cuivre, et par conséquent faire des observations magnétiques, même quand la mer sera fortement agitée. » (BSGP, 1825, S1, T3 : 150-151)

"Se garantir des erreurs", voilà bien tout l'enjeu qui réside dans l'utilisation des instruments et dans leur perfectionnement incessant. Mais la technique n'est rien sans sa maîtrise. Pour s'assurer en effet que l'usage des instruments participe du dispositif de la preuve qui se met alors en place, l'encadrement des gestes des géographes se fait plus présent. La société parisienne se pose en arbitre du geste géographique, conseillant les meilleures pratiques, stigmatisant les mauvaises et, ce faisant, édictant ainsi un code de scientificité des gestes du géographe. En 1825, elle conseille par exemple sur le meilleur usage du sextantpour des relevés topographiques plus efficaces (BSGP, 1825, S1, T3).

Cette mise en œuvre d'une scientificité accrue, du moins posée comme un horizon d'attente, s'articule à l'activité du voyage ${ }^{9}$. Au cours des décennies 1820 et 1830 , la professionnalisation de l'activité géographique passe en grande partie par l'affirmation du relevé d'informations comme étape essentielle, voire incontournable, de la chaîne d'information géographique. Dans le premier bulletin de la Société de Géographie de Paris, l'importance des voyages est immédiatement soulignée, ainsi que les moyens que la société souhaite développer pour les encourager :

«Indiquer les projets de voyage les plus faciles à exécuter, les moins dispendieux, et les plus susceptibles d'un résultat utile; en calculer les frais, en prévoir les difficultés, en spécifier les moyens d'exécution; trouver les hommes capables de se charger de ces entreprises; indiquer, au défaut de voyageurs, les diverses classes d'observateurs qui, fixés dans un lieu, seraient à portée de faire des recherches sur les objets qui, dans leurs environs, intéressent la Géographie, ou qui seraient disposés à résoudre les questions que la Société leur adresserait ; enfin, au défaut de voyageurs et d'observateurs locaux, indiquer les lacunes de la Géographie qui peuvent être remplies par des travaux de cabinet et des recherches d'érudition. » (BSGP, 1822, S1, T1 : 6)

Combler les «lacunes", ou les blancs de la carte pour le dire autrement (LaboulaisLesage, 2004), voilà l'objectif principal des sociétés de géographie, qu'elles transmettent à tous ceux qui participent à son activité. Et, même si le travail de cabinet peut le satisfaire en dernier ressort, même si d'ailleurs certains considèrent avec dédain le temps de l'exploration, le régime de scientificité de la géographie doit reposer sur la preuve, recueillie in situ. Pour cela, la société de Paris se fait une grande promotrice d'expéditions et voyages(voir annexe 1), et en pose les cadres scientifiques. Dès le second bulletin, elle suggère une série de questions aux voyageurs par région géographique du monde, afin d'orienter leurs travaux, dans un recueil intitulé 
Questions proposées aux voyageurs et à toutes les personnes qui s'intéressent aux progrès de la géographie :

« Non seulement un recueil de questions imprimées est le moyen le plus commode pour communiquer à tous les voyageurs, à tous observateurs les idées, les vœux de la Société ; non seulement, ce mode de correspondance, en assurant de plus grands succès que l'envoi par manuscrit, n'entraînera guères un surcroît réel de dépenses; mais il aura encore le résultat de faire naître, par la réunion successive des cahiers, un ouvrage d'une haute utilité pour les savants et les voyageurs, un monument scientifique sur lequel chaque membre de la Société pourra inscrire son nom et qui, même dans le cas d'un résultat peu favorable, attestera au monde savant les vues éclairées qui dirigent votre association. » (BSGP, 1824, S1, T2 : 71)

Ces précautions n'empêchent pas bien sûr la pluralité de pratiques individuelles, mais elles signalent la volonté de la société d'encadrer et normaliser les pratiques, dans le souci d'assurer l'universalité et la comparabilité des savoirs géographiques.

39 Car, au-delà des gestes et des pratiques, l'exigence générale est celle de l'écriture scientifique des savoirs géographiques, l'argumentaire devant relever de la démonstration et de la preuve. Quelques exemples de formulation puisés chez Jomard éclairent cette tonalité d'écriture :

40 "Puisque nous ignorons presque tout encore sur les courans (sic) qui coulent vers le centre de l'Afrique septentrionale, nous devons partir du Nil inférieur, où la science a pénétré avec ses instruments. De là nous remonterons de proche en proche jusqu'au point où nous pourrons parvenir. » (Jomard, $1825: 5$ )

41 " Il ne paraît donc pas exister à l'occident et à cette distance du royaume du Bornou, de montagnes élevées comparables à celles de l'Atlas, ni même de hauteurs dignes d'être mentionnées; et par conséquent, il ne peut en sortir de rivières dont le niveau soit assez élevé pour qu'elles s'écoulent dans le Nil. » (Ibid : 19)

Ces deux citations font ressortir quelques grands principes de scientifisation de l'activité et, partant, du discours géographique : objectivation des pratiques et des méthodes, progressivité et cumulativité des faits géographiques, comparabilité des savoirs. Tout cela prend place dans l'ambition générale des géographes du début du XIXe siècle de fonder une science fiable, sérieuse et légitime. Cette recherche de scientificité participe de la volonté de produire des savoirs universels, produits par tous et pour tous.

\section{Les limites d'une réflexivité grandissante}

Si la volonté d'assurer à la géographie une scientificité solide grandit au début du siècle, le regard porté sur le programme scientifique de la géographie est, quant à lui,encore très maigre, du moins jusqu'aux années 1840. L'ambition première semble en effet de « faire discipline » avant de «faire science ", alors même que la scientificité est placée au cœur du projet.

Considérant les quelques dictionnaires consacrés aux termes géographiques, plusieurs remarques s'imposent. Tout d'abord, ceux-ci sont quasiment inexistants jusqu'au milieu du siècle ou réduits à des glossaires sommaires au sein de monographies (Péaud, 2016). Quand ce type d'ouvrage existe, les définitions données se caractérisent par leur 
grande brièveté. Prenons l'exemple de la division de la terre en continents. Du côté deVosgien, on a :

«La terre se divise en continens (sic) et en îles. Le continent, appelé aussi terre ferme, est une grande partie de la terre qui comprend plusieurs régions qui ne sont point séparées les unes des autres par la mer. / Il y a deux grands continens, l'ancien et le nouveau : l'ancien comprend l'Europe, l'Asie et l'Afrique : sa plus grande longueur se mesure depuis le nord de la Tartarie orientale jusqu'au cap de Bonne-Espérance; elle est d'environ 3600 lieues. / Le nouveau continent porte le nom d'Amérique, sa plus grande longueur doit être prise du détroit de Magellan jusqu'au vieux Groënland. / Une île est une terre plus petite que le continent, entourée d'eau de tous côtés. / Dans le continent et dans l'île il y a cinq choses à remarquer, qui sont les presqu'îles, les isthmes, les caps, les montagnes, et les côtes. » (Vosgien, $1825: 3$ )

Du côté britannique, voici ce qui est indiqué à l'article « continent »chez Conder par exemple $(1834,172)$ :

47 " Continent. A vast tract of continuous land. Taken in the largest sense as used by geographers, the word denotes the two great portions of the earth's surface, sometimes called the old and the new world; the one comprising Asia, Africa, and Europe, the other both Americas. Australia or New Holland is sometimes called a third continent form its immense size, but with doubtful propriety, not being an assemblage of countries, but strictly a distinct island. $»^{10}$ (Conder, $\left.1834: 172\right)$

La notion de continent, prise comme une partie du globe, apparaît très floue pour les deux auteurs, dans ses caractéristiques physiques : il ne s'agit que d'une grande portion d'espace. Mais quelle taille cela recouvre-t-il ? Quelles réalités physiques ? Dans quelle proportion est-ce différent d'une île, que Vosgien amène en contrepoint? Les descriptions se caractérisent par une certaine évanescence, que Louis Marrou a bien analysé pour les îles (Marrou, 1998), dont les critères de définition se fixent tardivement. Bernard Debarbieux a aussi fait ce travail concernant la notion de «montagne» chez Alexander von Humboldt et montré l'indigence de sa conceptualisation (Debarbieux, 2012). Cela s'explique par le souci prépondérant de combler les lacunes en termes de connaissance du monde, c'est-à-dire de nommer et de recenser les objets géographiques, de comprendre les processus, avant d'analyser et de conceptualiser les modalités de production des savoirs.

Les géographes prennent conscience à partir des années 1840 de la nécessité de faire également science, c'est-à-dire justement de doter la géographie d'un programme, en passant de la recension et de la description à l'analyse. De la même façon qu'ils engagent une plus grande réflexivité sur leurs pratiques et leurs méthodes, ils expriment une exigence accrue de théorisation et de conceptualisation du discours géographique. Le duo Edme-François Jomard et Julian Jackson ${ }^{11}$ se situe aux avantpostes de cette réflexion. En 1847, ils pointent les améliorations à effectuer :

50 «Les noms génériques donnés aux différentes formes des continents et des mers auraient besoin eux-mêmes de passer par une révision sévère, et d'être soumis à une définition exacte, surtout leur application aux divers lieux de la terre." (Jomard, in BSGP, 1847, S3, T7 : 256-257)

51 «Il n'y a plus de très grandes découvertes à faire, bien que de nombreux détails manquent encore pour compléter la connaissance de l'intérieur de l'Afrique, du centre de l'Asie et du sud de l'Amérique ; avec le temps, on obtiendra ces notions, et le tracé 
cartographique de la terre deviendra assez complet, autant du moins qu'il est permis de l'espérer, pour remplir les blancs qui existent encore et pour rectifier graduellement ce qui pourrait encore se trouver inexact. Ce dont nous avons maintenant besoin, c'est de construire un bel et complet édifice avec les abondants matériaux réunis jusqu'à ce jour. » (Jackson, in BSGP, 1847, S3, T8 : 81)

Ces deux extraits en disent long sur les progrès réalisés et à effectuer. Tout d'abord, Jackson défend une nouvelle vision de la discipline, qui ne serait plus uniquement basée sur l'actualisation des données, mais qui construirait une représentation analytique du monde. Ce changement de considération de la géographie est loin d'être anodin, car la discipline se construit précisément sur son rapport au présent et sa valeur repose presque exclusivement dans la prise en charge de l'actualité du monde. La proposition de Jackson formule une rupture dans le temps de la discipline: son édification s'est faite dans le but de "remplir les blancs » des cartes, mais "maintenant», et cet adverbe invite à un tournant radical, il s'agit de mettre en ordre ce qui a été recueilli jusque-là. Les savoirs géographiques ne sont, de fait, plus considérés uniquement comme le résultat brut de recueil de données à travers le globe, mais bien celui d'une mise en ordre savante, critique et intelligente du monde, dans le but de le rendre intelligible. Cette rupture n'est cependant pas possible sans un réel effort conceptuel. Le tableau dressé est sans appel : il faut procéder à une "révision sévère ", autrement dit étayer enfin le contenu de ces termes génériques dont le sens n'est, si on lit entre les lignes, pas universellement et communément assuré.

Malgré cette volonté, ce souci demeure encore très largement marginal et peu entendu au milieu du XIXe siècle. La variété des approches et l'urgence d'assurer une visibilité disciplinaire à la géographie, qui est bientôt doublée du rôle accordé à la discipline dans la défense des intérêts nationaux (1860-1940), expliquent la difficulté et la frilosité de s'engager dans la construction collective d'une véritable épistémè géographique et explique en partie pourquoi la discipline demeure au stade, du moins en France, de la nomenclature et de la description. Le paradoxe d'une discipline sans programme scientifique ne sera résolu, pour la géographie française, qu'avec les propositions vidaliennes à la toute fin du siècle, lorsque la reconnaissance publique de la géographie s'appuiera sur un projet épistémologique.

\section{Conclusion}

L'élan géographique qui s'exprime au début du XIXe siècle en France et la première tentative d'institutionnalisation de la discipline qui l'accompagne traduisent donc moins la création d'un programme scientifique (Lakatos, 1994) que la volonté de rendre publique la géographie et de la faire exister comme discipline dans un paysage scientifique national et européen en recomposition. L'envie de "faire discipline », qui émerge chez ceux qui se reconnaissent progressivement comme géographes, se heurte à la difficulté de fonder conceptuellement, théoriquement mais aussi méthodologiquement les cadres d'une pensée géographique du monde. La disciplinarisation de la géographie reste donc partielle au début du XIXe siècle, s'appuyant presque exclusivement sur un lieu et quelques figures, mais ne parvenant pas à imposer la géographie dans l'ensemble du paysage scientifique français. En effet, elle reste pour cette période éloignée des lieux d'enseignement et propose un programme scientifique relativement indigent, parce que, par ailleurs, son objectif se 
situe dans un relevé nomenclatural des phénomènes et processus. Prenant peu à peu conscience de cette dernière difficulté, certains géographes plaident au milieu du siècle pour le développement d'une géographie analytique, et non plus seulement descriptive. Cependant, la variété des projets et la fragilité encore marquée du collectif géographique, en dépit des réels efforts d'institutionnalisation, empêchent d'aboutir à une épistémè géographique solide. Le tournant s'opère dans la dernière moitié du XIXe siècle, au moment où convergent le développement de l'enseignement géographique à tous les niveaux et l'élaboration d'un programme scientifique, c'est-à-dire quand faire discipline signifie aussi faire science.

\section{BIBLIOGRAPHIE}

Beck H., 1979,Carl Ritter. Genius der Geographie, Berlin, Dietrich Reimer Verlag.

Besse J.-M., 2003,Face au monde. Atlas, jardins, géoramas, Paris, Desclée de Brouwer, collection «Arts et esthétique».

Besse J.-M., Blais H. et Surun I., 2010,Naissances de la géographie moderne, ENS Editions, Lyon 2010.

Bret P., 2003, « L'Égypte de Jomard : la construction d'un mythe orientaliste, de Bonaparte à Méhémet-Ali », Romantisme, 2003, 120, 5-14.

Bret P., 1991, « Le Dépôt de la Guerre et la formation scientifique des ingénieurs-géographes militaires en France (1789-1830) », Annals of Science, vol. 48, 2, 113-157.

Blais H., Laboulais I. (dir.), 2006,Géographies plurielles - les sciences géographiques au moment de l'émergence des sciences humaines (1750-1850), Paris, L'Harmattan, coll. Histoire des sciences humaines.

Bourguet M.-N., Licoppe C. et Sibum O., 2002, Instruments, travel and science : itineraries of precision from the seventeenth to the twentieth century, London - New York, Routledge.

Calbérac Y., 2010, Terrains de géographes, géographes de terrain. Communauté et imaginaire disciplinaires au miroir des pratiques de terrain des géographes français au XXème siècle, Université Lumière Lyon 2, 2010.

Conder J., 1834, Dictionary of geography, ancient and modern, London, T. Tegg.

Debarbieux B., 2012, » The various figures of Mountains in Humboldt's Science and Rhetoric », Cybergeo : European Journal of Geography,doi :10.4000/cybergeo.25488.

Godlewska A., 1991, "L'influence d'un homme sur la géographie-française : Conrad Malte-Brun (1775-1826)”, Annales de géographie, 558, 191-206.

Godlewska A., 1999, French geographic science from Cassini to Humboldt, Chicago, University of Chicago Press.

Grataloup C., 2007,Géohistoire de la mondialisation. Le temps long du monde, Paris, Armand Colin, collection $\mathrm{U}$. 
Humboldt A., 1836-1837,Histoire de la Géographie du Nouveau Continent et des Progrès de l'Astronomie Nautique aux Quinzième et Seizième Siècles, Paris, Gide, 3 vol.

Jomard E.-F., 1831,Considérations sur l'objet et les avantages d'une collection spéciale consacrée aux collections géographiques et aux diverses branches de la géographie, Paris,Duverger.

Jomard E.-F., 1825, Extrait d'un mémoire sur la communication du Nil des Noirs ou Niger avec le Nil d'Egypte, Académie Royale des Sciences.

Jomard E.-F., 1848,De la Collection géographique créée à la Bibliothèque royale, examen de ce qu'on a fait et de ce qui reste à faire pour compléter cette création et la rendre digne de la France, Paris, Duverger.

Jomard E.-F., 1862,Les monuments de la géographie, Paris, Duprat, Franck, Arthus Bertrand, Renouard, Gide et Baudry, Delion, 21 planches.

Laoulais-Lesage I. (dir.), 2004,Combler les blancs de la carte. Modalités et enjeux de la construction des savoirs géographiques (XVIè-XXè siècle), Strasbourg, Presses Universitaires de Strasbourg.

Laboulais-Lesage, 2005, «Les géographes de l'Encyclopédie méthodique », in : Blanckaert C. et Porret M. (dir.),L'Encylopédie méthodique (1782-1832). Des Lumières au positivisme, Droz, Genève.

Lakatos I., 1994,Histoire et méthodologie des sciences : programme de recherche et reconstruction rationnelle, Paris, Presses Universitaires de France.

Laissus Y., 2004,Jomard, le dernier égyptien, Paris, Fayard.

Lefort I., 1990, La lettre et l'esprit, géographie scolaire, géographie savante, 1870-1970, Paris, Presses du CNRS.

Lejeune D., 1993,Les sociétés de géographie en France et l'expansion coloniale au XIXème siècle, Paris, Albin Michel.

Lüdecke C., 2002, Carl Ritters Lehrtätigkeit an der Allgemeinen Kriegsschule in Berlin (1820-1853), Berlin, Verlag für Wissenschafts- und Regionalgeschichte.

Malte-Brun C., 1807, Annales des Voyages, de la Géographie et de l'Histoire, Paris, Chez Buisson, Tome Premier.

Marrou L., 1998, » La Macaronésie et les Géographies Universelles Françaises », Norois, 1998, vol. 180, no 1, pp. 667-684.

Nordman D., 1994,L'Ecole Normale de l'an III. Leçons d'histoire, de géographie, d'économie politique. Edition annotée des cours de Volney, Buache de La Neuville, Mentelle et Vandermonde avec introductions et notes (directions de l'ensemble du volume « Introduction générale. Les sciences morales et politiques à l'Ecole Normale », et édition des « Leçons de géographie de Buache et Mentelle » en collaboration avec François Labourie), Paris, Dunod, in- $4^{\circ}$.

Péaud L., 2016,La géographie, émergence d'un champ scientifique. France, Prusse et GrandeBretagne, ENS Éditions, Lyon.

Santarem M.F., 1842, Recherches sur la priorité de la découverte des pays situés sur la côte Occidentale d'Afrique, au-delà du cap Bojador, et sur les progrès de la science qéographique, après les navigations des portugais au XVe siècle, accompagnées d'un atlas composé de mappemondes, et de cartes pour le plupart inédites, dressées depuis le XIe siècle jusqu'au XVIIe siècle, Paris, Fain et Thunot. 
Société de géographie de Paris, 1825-1899 Bulletin de la Société de géographie de Paris, consulté le 24 avril 2017, URL : http://gallica.bnf.fr/ark:/12148/cb34424377d/date.

Thiesse A.-M., 1999, La création des identités nationales, Europe XVIIIème-XXème siècle, Paris, L'Univers Historique/Seuil.

Vosgien M., 1825,Dictionnaire géographique ou Description de toutes les parties du monde, Nouvelle édition précédée d'un précis de géographie par J. D.Goigoux, Paris, Ménard et Dessenne.

\section{ANNEXES}

Annexe : Les prix proposés par la Société de Géographie de Paris entre 1821 et 1830 (source : BSGP)

\begin{tabular}{|c|c|}
\hline Année & Sujets et thèmes \\
\hline 1823 & $\begin{array}{l}\text { Déterminer la direction des chaînes de montagnes de } \\
\text { l'Europe, leurs ramifications et leurs élévations } \\
\text { successives dans toute leur étendue }\end{array}$ \\
\hline $\begin{array}{l}\text { Rechercher l'origine des divers peuples } \\
\text { répandus dans les îles du Grand Océan }\end{array}$ & \\
\hline $\begin{array}{l}\text { Itinéraire statistique et commercial de } \\
\text { Paris au Hâvre de Grâce }\end{array}$ & \\
\hline $\begin{array}{l}\text { Analyse des ouvrages de géographie et } \\
\text { de statistique, récemment publiés en } \\
\text { langue russe }\end{array}$ & \\
\hline 1824 & $\begin{array}{l}\text { Tracer une carte de la Guyane Française, en donnant une } \\
\text { description géographique }\end{array}$ \\
\hline $\begin{array}{l}\text { Encouragement pour voyage en Afrique, } \\
\text { en particulier dans la Nouvelle } \\
\text { Cyrénaïque }\end{array}$ & \\
\hline $\begin{array}{l}\text { Description complète des régions } \\
\text { naturelles de la France }\end{array}$ & \\
\hline 1825 & Voyage à Tombouctou \\
\hline $\begin{array}{l}\text { Voyage dans la partie méridionale de la } \\
\text { Caramanie (Lycie, Pamphylie et Cylicie) }\end{array}$ & \\
\hline 1826 & $\begin{array}{l}\text { Nivellements barométriques les plus étendus faits sur les } \\
\text { lignes de partage des eaux des grands bassins de la } \\
\text { France }\end{array}$ \\
\hline $\begin{array}{l}\text { Etude de la côte méridionale de la } \\
\text { Manche }\end{array}$ & \\
\hline
\end{tabular}




\begin{tabular}{|c|c|}
\hline $\begin{array}{l}\text { Etude des antiquités américaines (village } \\
\text { de Palenqué) }\end{array}$ & \\
\hline 1827 & Prix annuel : Nivellement de la France \\
\hline Exploration de l'intérieur de la Guyane & \\
\hline $\begin{array}{l}\text { Exploration de la Caramanie et de } \\
\text { l'Himalaya }\end{array}$ & \\
\hline $\begin{array}{l}\text { Voyage dans ancienne Babylonie et la } \\
\text { Chaldée }\end{array}$ & \\
\hline Voyage à l'ouest du Darfour & \\
\hline 1828 & $\begin{array}{l}\text { Prix annuel: La plus grande découverte annuelle en } \\
\text { géographie }\end{array}$ \\
\hline $\begin{array}{l}\text { Prix annuel : Communication des } \\
\text { notions utiles à la géographie }\end{array}$ & \\
\hline 1829 & Exploration de la région Marawi \\
\hline 1830 & Origine des populations nègres asiatiques \\
\hline
\end{tabular}

\section{NOTES}

1. Il n'est pas question au début du $19^{\mathrm{e}}$ siècle de constituer une école française de géographie, comme ce sera le cas à la fin du siècle autour de Paul Vidal de la Blache. Aussi parlons-nous ici de la tentative qui a lieu en France, sans confondre ce projet avec une volonté de le nationaliser. Au contraire, ceux qui se reconnaissent dans la géographie sont bien davantage portés par une volonté universaliste (Péaud, 2016). 2. Abrégé en «BSGP » par la suite.

3. Les géoramas sont des dispositifs en trois dimensions, sphériques, qui représentent la Terre et son relief. Généralement creux, ils se visitent et donnent à voir la Terre. Ils connaissent dans les années 1840-1850 un franc succès en France et en Angleterre. Voir : Besse Jean-Marc, 2003, Face au monde. Atlas, jardins, géoramas, Paris, Desclée de Brouwer, collection "Arts et esthétique", $244 \mathrm{p}$.

4. Avant 1870, une seule chaire de géographie historique existe à la Sorbonne (créée en 1812), elle est occupée dans les années 1860 et 1870 par Auguste Himly, chargé avec Emile Levasseur de la réforme de l'enseignement géographique (Lefort, 1990).

5. Les membres de la Société de Géographie de Paris se caractérisent encore, pour la plupart, par leur encyclopédisme et donc un investissement scientifique non spécifique et non exclusif à la géographie. Mais ils font de leurs rencontres à la Société de Paris l'occasion d'entretenir une sociabilité aristocratique et bourgeoise (Lejeune, 1993).

6. En 1828, Caillé atteint Tombouctou et décroche ainsi le prix proposé par la Société de géographie de Paris. Jomard soutient son succès et défend la primauté de son entreprise face aux récriminations anglaises. 
7. D’autres géographes arabes sont parfois mentionnés, tels que Ibn Khordadbeh et Ibn Hawqal, dans leurs liens avec Al Idrisi.

8. Il est d'autant plus intéressant de noter que les géographes du XIXe siècle s'appuient sur un temps de découvertes et d'explorations pour asseoir leur géographie sur une pratique de plus en plus marquée du terrain et de l'arpentage (Péaud, 2016). L'exploitation de cet effet de continuité n'est sans doute pas anodine dans leur entreprise de justification.

9. Nous n'employons pas ici le terme «terrain », anachronique au début du XIX ${ }^{\mathrm{e}}$ siècle (Calbérac, 2010). Les géographes parlent eux-mêmes de « voyage » ou d' « exploration », dont le statut scientifique n'est pas aussi assuré que le sera le terrain pour les géographes vidaliens, par exemple.

10. Traduction : "Continent. Une vaste étendue de terre continue. Pris dans un sens large tel qu'utilisé par les géographes, le mot indique les deux grandes portions à la surface de la terre, parfois appelées l'ancien et le nouveau monde ; le premier comprend l'Asie, l'Afrique et l'Europe, l'autre les deux Amériques. L'Australie ou la Nouvelle-Hollande est parfois considérée comme un troisième continent de par son immense taille, mais avec des propriétés douteuses, car n'étant pas un assemblage de pays, mais strictement une île distincte. » 11. Julian Jackson est membre de la Royal Geographical Society de Londres. Il correspond très souvent avec Jomard sur les ressorts proprement épistémologiques de la géographie.

\section{RÉSUMÉS}

Au début du XIXe siècle, la géographie n'existe pas en tant que telle dans le paysage scientifique français ou européen. Pourtant, certains individus commencent à se mobiliser pour en faire une discipline, ayant le même statut que l'histoire naturelle ou l'histoire. Cette ébauche d'institutionnalisation s'appuie sur la fondation d'un collectif géographique, se retrouvant dans des lieux et des figures incarnant la discipline. Malgré cet élan, les premiers géographes ne parviennent pas à faire science, car l'ambition touche davantage la reconnaissance publique de la discipline que la constitution d'un véritable programme scientifique. En s'intéressant au contexte français entre 1800 et 1850 , cet article vise donc à questionner un moment peu connu et peu interrogé de l'histoire de la géographie, celui où un élan disciplinaire s'exprime, et à mettre en avant les modalités épistémologiques d'une première tentative d'institutionnalisation.

At the beginning of the 19th century, in France or in Europe, geography does not exist as a discipline in the scientific field. However, some figures start to show geography as a discipline and behave to act, so that geography can compete with natural history or history. This first institutionalisation is based on the foundation of a geographical community, which can meet in places and identify itself in key figures. Despite this desire, the first geographers do not create a real scientific program for geography. Their search for recognition allows them to partially found a discipline, but not a science. Regarding the French situation between 1800 and 1850, this paper aims to question an unknown moment in the history of geography, one of the first rushes for a geographical discipline and examines the epistemological ways of this movement. 
INDEX

Thèmes : Carnets de recherches

Mots-clés : histoire de la géographie, France, discipline, géographie, institutionnalisation scientifique, XIXe siècle, société de géographie

Keywords : History of Geography, France, Discipline, Geography, Scientific Institutionalisation, 19th Century, Society of Geography

\section{AUTEUR}

\section{LAURA PÉAUD}

Maître de conférences

Laboratoire PACTE (UMR 5194)

Université Grenoble Alpes

laura.peaud@univ-grenoble-alpes.fr 